\title{
An Optical Fiber Hydrophone Using Equivalent Phase Shift Fiber Bragg Grating for Underwater Acoustic Measurement
}

\author{
Shengye HUANG ${ }^{1}$, Xiaofeng JIN ${ }^{1}$, Jun ZHANG ${ }^{2}$, Yi CHEN ${ }^{2}$, \\ Yuebin $\mathrm{WANG}^{2}$, Zhijun $\mathrm{ZHOU}^{1}$, and Juan $\mathrm{NI}^{1}$ \\ ${ }^{1}$ Department of Information Science \& Electronic Engineering, Zhejiang University, Hangzhou, 310027, China \\ ${ }^{2}$ Hangzhou Applied Acoustics Research Institute, Hangzhou, 310014, China \\ *Corresponding author: Xiaofeng JIN_E-mail: Jinxf00@zju.edu.cn
}

\begin{abstract}
An optical fiber hydrophone based on equivalent phase shift fiber Bragg grating (EPS-FBG) with temperature compensation package provides an improvement of sensitivity in underwater acoustic measurement at wide frequency range, from $2.5 \mathrm{kHz}$ to $12 \mathrm{kHz}$. The acoustic pressure is transduced into elastic vibration of a circle metal disk, resulting in an intensity modulation of the reflected light wave back from fiber Bragg grating (FBG). Experiment shows that the packaged EPS-FBG hydrophone has a minimum detectable acoustic pressure of about $500 \mu \mathrm{Pa} / \sqrt{\mathrm{Hz}}$ at $5 \mathrm{kHz}$ and achieves about $18-\mathrm{dB}$ improvement of acoustic pressure sensitivity compared with a regular apodized FBG hydrophone.
\end{abstract}

Keywords: Optical fiber hydrophone, acoustic pressure sensitivity, equivalent phase shift fiber Bragg grating, temperature compensation package

\section{Introduction}

There exists a great demand for measurement of underwater acoustic signal in many applications, e.g. constructions, geophysical and military fields [1-2]. Conventional detection was usually preformed with piezoelectric devices, such as piezoceramics and piezoelectric polymers [3-4]. Compared with the traditional piezoelectric hydrophone, fiber optic hydrophones, which were first reported by Bucaro et al. in 1977, are distinguished for their flexibility, light sensor weight, low hydrophone cost, immunity to electro-magnetic interference (EMI) and the property that all active electronics can be kept out of water [5-6]. Interferometric hydrophones, as a main branch of fiber optic hydrophones, have already been applied in some fields [7-8]. Nowadays, with the prevalence of fiber Bragg gratings (FBGs), many new advantages such as smaller size, reliability and stability in severe environment, multiplexing capability based on wavelength division multiplex (WDM) all have been introduced into FBG-based hydrophones [9-11]. The operating principle of FBG-based hydrophone is typically based on intensity modulation of light wave, caused by instant change of the transmission spectrum of sensor under the influence of acoustic field [12]. However, low sensitivity to acoustic pressure will cause that FBGs seemingly may not satisfy the high sensitivity requirement of hydrophones [13]. In this paper, a kind of simplified hydrophone package structure with improved sensitivity, good linearity, and isotropic directivity is proposed based on equivalent phase shift fiber Bragg grating (EPS-FBG).

Received: 27 October 2010 / Revised version: 5 December 2010

(C) The Author(s) 2011.This article is published with open access at Springerlink.com 
Section 2.1 describes the structure of EPS-FBG hydrophone. The transduction mechanism of probe is described in Section 2.2. Experimental setup is provided in Section 3.1, and the acoustic performance of the hydrophone in terms of time-domain performance, sensitivity, and directivity is discussed in Section 3.2.

\section{Fiber Bragg grating hydrophone}

\subsection{Hydrophone structure and fabrication}

The inside structure of packaged EPS-FBG hydrophone is illustrated in Fig. 1. The EPS-FBGs were achieved by changing sampling period as described in [14-15]. The EPS-FBGs were fabricated in our experiment by using a ultraviolet (UV) excimer Braggstar 200 operating at $248 \mathrm{~nm}$. An EPS-FBG with length of about $2 \mathrm{~cm}$ was used for the assembly of EPS-FBG hydrophone. The bare fiber at two ends of EPS-FBG was attached to a small copper pipe separately with epoxy. With the help of copper pipes and epoxy, one end of EPS-FBG was attached to the center hole of a thin metal disk, which was laser-welded on the upper mouth edge of a cylindrical alloy shell. The other end of EPS-FBG was attached to the bottom outlet of the shell. The EPS-FBG in the center housing was initially kept at a fixed tension during packaging. The circle metal disk with thickness of $0.1 \mathrm{~mm}$ functioned as a pressure transducer for enhancing the dynamic sensitivity of the EPS-FBG. The temperature insensitive operation was achieved in our packaged structure. The mechanism for temperature compensation is same as that in [16]. The cylindrical shell made of invar alloy was used as the low thermal expansion component, and two small copper pipes in Fig. 1 were used as the compensating members. If a change of temperature occurs, the copper pipes expand or contract in order to cancel out the center wavelength shift of hydrophone due to the change of temperature. In this way, we can make packaged FBG hydrophones with extremely low temperature dependence.

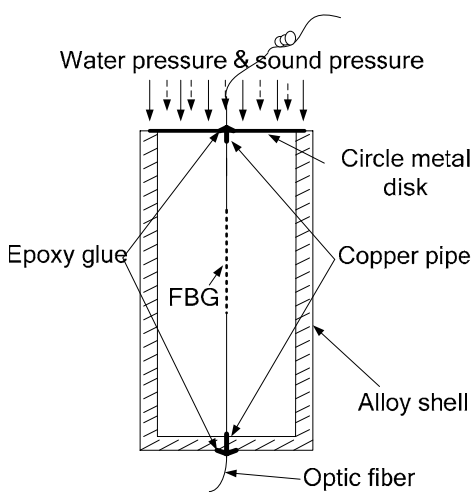

Fig. 1 Schematic of EPS-FBG hydrophone.

\subsection{Principles of operation}

\subsubsection{Acoustic transduction mechanism}

A simplified representation of FBG hydrophone as a sensing element in operation is shown in Fig. 2. An incoming acoustic pressure working on the circle metal disk, will force it to oscillate along its normal. And then the FBG will also have an axial oscillation transmitted from the disk. Owing to elasto-optic effect, this oscillation will induce a dynamic shift of power transmission spectrum of FBG caused by the incoming acoustic wave.

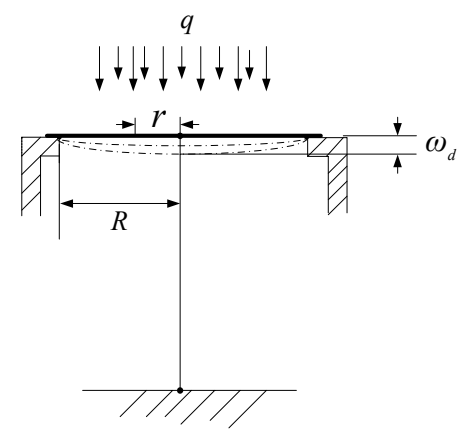

Fig. 2 A simplified representation of FBG hydrophone structure ( $q$ : pressure added to metal disk; $R$ : the radius of disk; $r$ : distance from some place on the disk to the center; $\omega_{d}$ : the deflection of disk)

\subsubsection{Sensitivity analysis}

The hydrophone probe can be equivalent to an ideal and concise system that only includes a circle metal disk and a FBG as shown in Fig. 2. The bending rigidity of the disk, $D$, is given by [17] 


$$
D=\frac{E_{d} t^{3}}{12\left(1-\mu^{2}\right)}
$$

where $E_{d}$ and $t$ are Young's modulus and thickness of metal disk, respectively, and $\mu$ is Poisson ratio.

When a pressure $q$, including water pressure and sound pressure, is added to disk-water interface evenly, the disk will have a deflection of $\omega_{d}$ :

$$
\omega_{d}(q, r)=\frac{q}{64 D}\left(R^{2}-r^{2}\right)^{2}
$$

where $R$ is the radius of the disk and $r$ is the distance from some place on the disk to the center. When the disk deforms, tension force $T$ will be transduced to the FBG, which is given by

$$
T=\varepsilon_{f} A E_{f}
$$

where $\varepsilon_{f}$ is the axial strain in the FBG, $A$ is the cross-sectional area of the FBG, and $E_{f}$ is Young's modulus of the FBG. The deflection caused by $T$ added at the center of the disk is

$$
\omega_{f}(T, r)=\frac{T}{16 \pi D}\left(\frac{3+\mu}{1+\mu}\left(R^{2}-r^{2}\right)+2 r^{2} \ln \frac{r}{R}\right) .
$$

The wavelength shift $\Delta \lambda_{\mathrm{B}}$ of the FBG is given by

$$
\Delta \lambda_{\mathrm{B}}=\lambda_{\mathrm{B}}(1-p) \varepsilon_{f}
$$

where $\lambda_{\mathrm{B}}=1550 \mathrm{~nm}$ is Bragg wavelength, $p=0.22$ is strain optic coefficient. Since $\varepsilon_{f}$ can be expressed as

$$
\varepsilon_{f}=\left[\omega_{d}(q, 0)-\omega_{f}(T, 0)\right] / L
$$

where $L$ is the length of fixed fiber. According to (2), (3), and (4), we can obtain the strain in the fiber:

$$
\varepsilon_{f}=\frac{\frac{q}{64 D} R^{4}}{L+\left(\frac{3+\mu}{1+\mu}\right) \frac{A E_{f}}{16 \pi D} R^{2}} .
$$

Consequently, the sensitivity of hydrophone can be obtained by

$$
\frac{\Delta \lambda_{\mathrm{B}}}{q}=\frac{\lambda_{\mathrm{B}}(1-p) \frac{R^{4}}{64 D}}{L+\left(\frac{3+\mu}{1+\mu}\right) \frac{A E_{f}}{16 \pi D} R^{2}} .
$$

From (8), we find $R, t, L$, and $E_{d}$ have significant effect on the sensitivity. The values of the parameters used in our experiment have been listed in Table 1.

Table 1 Parameters used in the FBG hydrophone.

\begin{tabular}{c|c}
\hline Parameter & Value \\
\hline$R$ & $3 \mathrm{~mm}$ \\
\hline$t$ & $0.1 \mathrm{~mm}$ \\
\hline$L$ & $4 \mathrm{~cm}$ \\
\hline$E_{d}$ & $106 \mathrm{GPa}$ \\
\hline$A$ & $0.0123 \mathrm{~mm}^{2}$ \\
\hline$E_{f}$ & $72 \mathrm{GPa}$ \\
\hline$\mu$ & 0.3 \\
\hline
\end{tabular}

We define the voltage-acoustic-pressure sensitivity as

$$
\frac{\Delta V}{q}=k \frac{\Delta \lambda_{\mathrm{B}}}{q} d_{g}
$$

where $k$ is a factor associated with the response of photo diode (PD) and normalized optical power, $d_{g}$ is the edge dynamic response of FBG, which is proportional to the gradient value of FBG spectrum at certain operating wavelength.

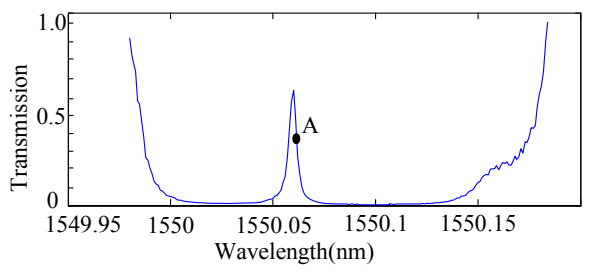

(a)

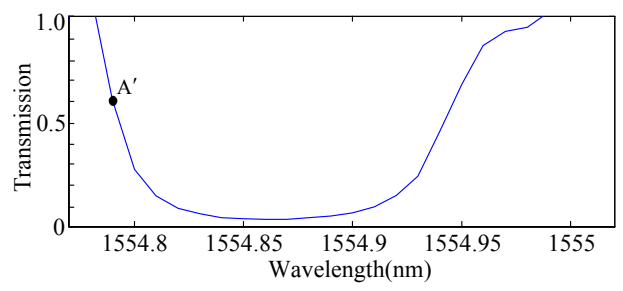

(b)

Fig. 3 Comparison of the optical transmission spectrum of (a) an EPS-FBG and (b) a regular apodized FBG.

The optical transmission spectrum of EPS-FBG used in the experiment is shown in Fig. 3(a). Its sampling period is $0.4 \mathrm{~mm}$ and sampling circle duty is 0.25 . Compared with a regular apodized FBG in Fig. 3(b), the advantage of using EPS-FBG is 
obvious. In our experiment, the gradient value of EPS-FBG spectrum at the operating point $\mathrm{A}$ in Fig. 3(a) is about 10 times as many as the one of regular apodized FBG at operating point $\mathrm{A}^{\prime}$ in Fig. 3(b). So the usage of EPS-FBG in FBG hydrophones is supposed to be able to improve acoustic pressure sensitivity of about $20 \mathrm{~dB}$.

\section{Experiment and discussion}

\subsection{Experimental setup}

A schematic diagram for testing the EPS-FBG hydrophone is shown in Fig. 4. The experiment was done in a water tank of $10 \mathrm{~m} \times 8 \mathrm{~m} \times 6 \mathrm{~m}$ in size, the sides of which were equipped with anechoic material. The low frequency limit caused by confined water tank was $2.5 \mathrm{kHz}$ for conventional pulsed-sound measurement technique. A lead zirconate titanate (PZT) acoustic transducer working as a sound projector was located at the middle of the water tank and a depth of $3 \mathrm{~m}$ for generating pulsed-sound signals. The EPS-FBG hydrophone under-test was placed at the same depth and $5 \mathrm{~m}$ 's away from the projector. The sound pressures at the hydrophone position were tested by a standard PZT hydrophone at frequency range of $2.5 \mathrm{kHz}$ to $12 \mathrm{kHz}$ at first. Light wave from the tunable laser source Agilent 81940A was injected into the EPS-FBG hydrophone through a fiber-optic circulator. The reflected light from the FBG was then routed to a photodiode and an optical power sensor Agilent 81635 A by a $1 \times 2$ optical coupler with power ratio 95:5. The photodiode converted the light wave into an electric signal, which was acquired by an oscilloscope. The data was transmitted to a personal computer (PC) by a general purpose interface bus (GPIB) cable for test and analysis of transient acoustic waveforms. The output of Agilent power sensor was monitored by the system for setting up operating wavelength. Agilent 81940A and Agilent $81635 \mathrm{~A}$ were equipped within a portable lightwave multimeter system Agiltent 8163A, which was connected to the PC through another GPIB cable. When the projector was silent, the shift of reflected power spectrum of FBG hydrophone at different water depths was tested by tuning the wavelength of Agilent 81940A laser source and monitoring the output of Agilent 81635A. In our system, the operating wavelength of FBG hydrophone could be set up dynamically by the computer in case of the center-wavelength shift resulting from the change of water depth or water temperature.

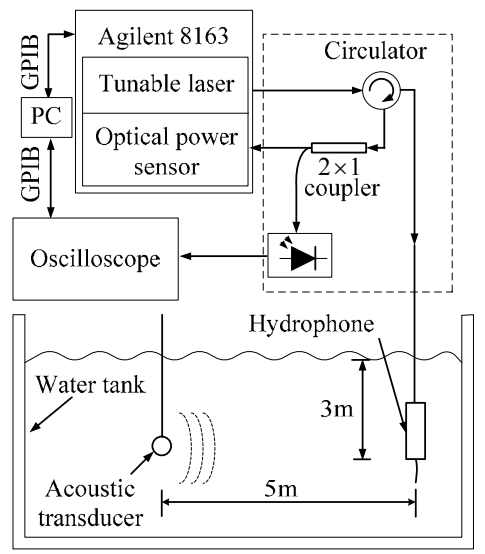

Fig. 4 Schematic diagram for testing the EPS-FBG hydrophone.

\subsection{Acoustic characteristics}

Figure 5 shows a temporal response of EPS-FBG hydrophone to a sound pressure pulse of $1 \mathrm{kPa}$ at the frequency of $5 \mathrm{kHz}$. From the fast Fourier transform (FFT) result of the temporal response data, we find the noise floor of the hydrophone is about $1 \mu \mathrm{V} / \sqrt{\mathrm{Hz}}$ at $5 \mathrm{kHz}$. Figure 6 shows the frequency response of EPS-FBG hydrophone. From Fig. 6, we obtain the average of tested sensitivit of $3.64 \mathrm{~nm} / \mathrm{MPa}$, which is a little better than the predicted value of $2 \mathrm{~nm} / \mathrm{MPa}$. The frequency response in Fig. 6 is not flat and has a vibrating peak because of the mechanical response of the packaged structure. According to the sensitivity and noise floor, the minimum detectable acoustic pressure at $5 \mathrm{kHz}$ with EPS-FBG hydrophone is about $500 \mu \mathrm{Pa} / \sqrt{\mathrm{Hz}}$ while it is estimated around $3500 \mu \mathrm{Pa} / \sqrt{\mathrm{Hz}}$ with a regular apodized FBG 
hydrophone.

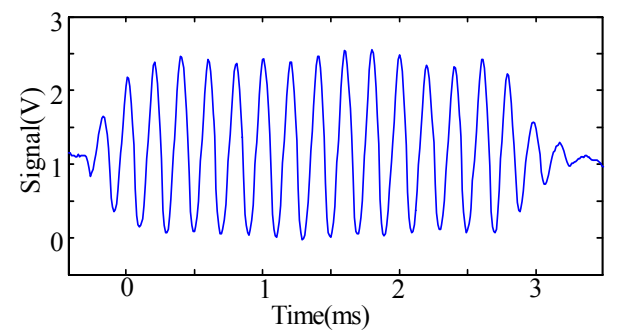

Fig. 5 Typical temporal response of EPS-FBG hydrophone to a sound pressure pulse of $1 \mathrm{kPa}$ at the frequency of $5 \mathrm{kHz}$.

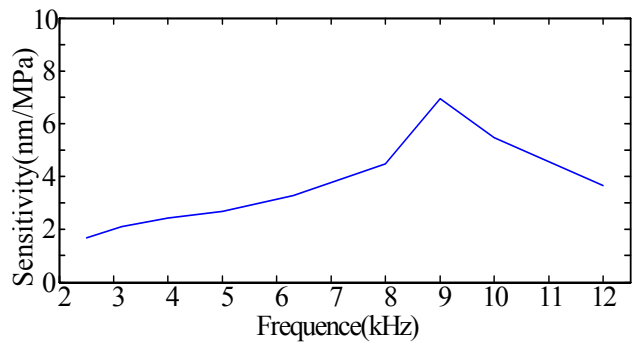

Fig. 6 Frequency response of EPS-FBG hydrophone.

In Fig. 7, curves (a) and (b) show the tested acoustic pressure sensitivity of the hydrophones based on the EPS-FBG and the regular apodized FBG respectively. The acoustic pressure sensitivity of the EPS-FBG hydrophone is approximately $18 \mathrm{~dB}$ higher than that of the regular one, which is consistent with the analysis of Section 2.2.

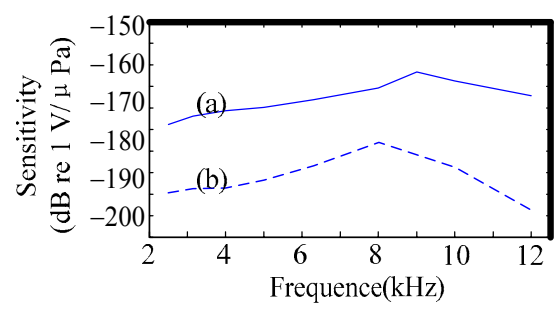

Fig. 7 Comparison of the voltage-pressure sensitivity between the FBG hydrophones using (a) an EPS-FBG and (b) a regular apodized FBG.

By uniformly rotating the hydrophone horizontally, we can get the directivity of acoustic sensitivity of the EPS-FBG hydrophone. The result is shown in Fig. 8 for the directivity of the hydrophone at acoustic signal frequency of $5 \mathrm{kHz}$, which demonstrates an isotropic behavior of the response.

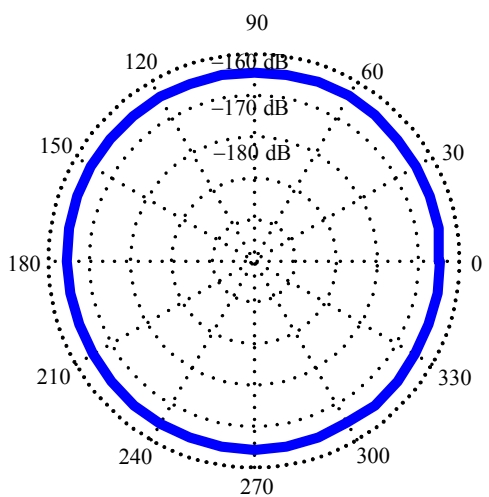

Fig. 8 Result of directivity measurement at a signal frequency of $5 \mathrm{kHz}$.

\section{Conclusions}

An optical hydrophone based on EPS-FBG is demonstrated in this paper. It features in high sensitivity, isotropic response, temperature independent, small package size, and convenience of cascading multiple hydrophones to form an acoustic array. Compared with regular apodized FBG, EPS-FBG hydrophone has significant improvement in acoustic pressure sensitivity. By optimizing the metal material and package size of the hydrophone, the sensitivity of the hydrophone can be efficiently tailored for some specific applications.

Open Access This article is distributed under the terms of the Creative Commons Attribution License which permits any use, distribution, and reproduction in any medium, provided the original author(s) and source are credited.

\section{References}

[1] Y. J. Rao, Y. P. Wang, Z. L. Ran, and T. Zhu, "Novel fiber-optic sensors based on long-period fiber gratings written by high-frequency $\mathrm{CO}_{2}$ laser pulses," J. Lightwave Technol., vol. 21, no. 5, pp. 1320-1327, 2003.

[2] H. Monma, N. Fujiwara, R. Iwase, K. Kawaguchi, S. Suzuki, and H. Kinoshita, "Monitoring system for submarine earthquakes and deep sea environment," in Proc. MTS/IEEE OCEANS'97, Halifax, NS, Canada vol. 2, pp. 1453-1459, 1997. 
[3] Y. Q. Yuan, B. W. Shi, and Z. J. Liu, "A new multilayer planar PVDF standard hydrophone and its applications," IEEE Trans. on Ultrason., Ferroelect., and Freq. Contr., vol. 42, no. 5, pp. 958-964, 1995.

[4] B. L. Jiao, "Investigation on piezoelectric helix for use as a hydrophone," IEEE Trans. on Ultrason., Ferroelect., and Freq. Contr., vol. 46, no. 6, pp. 1446-1449, 1999.

[5] J. A. Bucaro and H. D. Dardy, "Fiber-optic hydrophone," J. Acoust. Soc. Am., vol. 62, no. 5, pp. 1302-1304, 1977.

[6] G. A. Cranch, P. J. Nash, and C. K. Kirkendall, "Large-scale remotely interrogated arrays of fiber-optic interferometric sensors for underwater acoustic applications," IEEE Sensors J., vol. 3, no. 1, pp. 19-30, 2003.

[7] Z. F. Wang, Y. M. Hu, Z. Meng, and M. Ni, "Pseudo working-point control measurement scheme for acoustic sensitivity of interferometric fiber-optic hydrophones," Chin. Opt. Lett., vol. 6, no. 5, pp. 381-383, 2008.

[8] G. A. Cranch and P. J. Nash, "Large-scale multiplexing of interferometric fiber-optic sensors using TDM and DWDM," J. Lightwave Technol., vol. 19, no. 5, pp. 687-699, 2001.

[9] W. T. Zhang, F. Li, Y. L. Liu, and H. Xiao, "FBG hydrophone: theory and experiment," in Opt. Fiber Sensors Conf., 2008. APOS'08, 1st Asia-Pacific, Chengdu, Nov. 7-9, pp. 1-4, 2008.

[10] N. Takahashi, K. Yoshimura, S. Takahashi, and K. Imamura, "Development of an optical fiber hydrophone with fiber Bragg grating," Ultrasonics, vol. 38, no. 1-8, pp. 581-585, 2000.

[11] H. J. Sheng, M. Y. Fu, T. C. Chen, W. F. Liu, and S. S. Bor, "A lateral pressure sensor using a fiber Bragg grating," IEEE Photonics Technol. Lett., vol. 16, no. 4, pp. 1146-1148, 2004.

[12] A. Cusano, S. Campopiano, S. D’Addio, M. Balbi, S. Balzarini, M. Giordano, and A. Cutolo, "Plastic coated fiber Bragg gratings as high sensitivity hydrophones," in 5th IEEE Conf. on Sensors, Daegu, pp. 166-169, 2006.

[13] J. Yang, Y. Zhao, and X. J. Ni, "Development of novel fiber Bragg grating underwater acoustic sensor," Acta Optica Sinica, vol. 27, no. 9, pp. 1575-1579, 2007(in Chinese).

[14] Y. T. Dai, X. F. Chen, D. J. Jiang, S. Z. Xie, and C. C. Fan, "Equivalent phase shift in a fiber Bragg grating achieved by changing the sampling period," IEEE Photon. Technol. Lett., vol. 16, no. 10, pp. 2284-2286, 2004.

[15] X. Wang, C. X. Yu, Z. H. Yu, and Q. Wu, "Sampled phase-shift fiber Bragg gratings," Chin. Opt. Lett., vol. 2, no. 4, pp. 190-191, 2004.

[16] G. W. Yoffe, P. A. Krug, F. Ouellette, and D. A. Thorncraft, "Passive temperature-compensating package for optical fiber gratings," Appl. Opt., vol. 34, no. 30, pp. 6859-6861, 1995.

[17] S. Timoshenko and S. Woinowsky-Krieger, Theory of plates and shells. New York: McGral-Hill Book Company, 1959. 